\title{
HUMAN LEUKOCYTE ANTIGEN-B27 AND DISEASE SUSCEPTIBILITY IN VOJVODINA, SERBIA
}

\author{
Vojvodić $\mathrm{S}^{1}$, , Ademović-Sazdanić $\mathrm{D}^{1}$, Busarčević $\mathrm{I}^{2}$
}

\begin{abstract}
*Corresponding Author: Assistant Professor Svetlana Vojvodić, M.D., Ph.D., Department for Laboratory Testing, Tissue Typing Compartment, Institute for Blood Transfusion of Vojvodina, Hajduk Veljkova 9a, 21000 Novi Sad, Serbia; Tel.: +381-21-4877-963; Mobile: +381-64-861-58-12; Fax: +381-21-4877-978; E-mail:ssvu@EUnet.rs
\end{abstract}

\begin{abstract}
There are numerous studies showing the role of human leukocyte antigens (HLAs) related with susceptibility or resistance to certain diseases. The aim of this study was to determine the association of HLA-B27 with ankylosing spondylitis (AS), polyarthralgia, lumboishialgia, acute anterior uveitis (AAU), psoriatic arthritis (PA), synovitis coxae and rheumatoid arthritis (RA) in patients from Vojvodina, Serbia. An HLA I class typing was performed by the serological immunomagnetic two-color fluorescence method using peripheral blood $\mathrm{T}$ lymphocytes in 97 patients and 224 healthy controls from the population of Vojvodina, Serbia.We calculated HLA-B27 frequencies, relative risk (RR), ethiologic fraction (EF), e.g., population attributive risk, when RR was greater than 1, while, preventive fraction (PF) was calculated when RR was lower than 1 . This study revealed the strongest association of AS with HLA$\mathrm{B} 27$ antigen: $\mathrm{RR}=25.0$, while the $\mathrm{EF}$ was greater than 0.15 , respectively. The $\chi^{2}$ test showed the significant difference $(p<0.05)$ in HLA-B27 in patients with AS in comparison to controls $\left(\chi^{2}=52.5\right)$. It was concluded that there is a positive association of
\end{abstract}

\footnotetext{
${ }^{1}$ Department for Laboratory Testing, Tissue Typing Compartment, Institute for Blood Transfusion of Vojvodina, Novi Sad, Serbia

${ }^{2}$ University of Novi Sad, Medical Faculty Novi Sad, Novi Sad, Serbia
}

HLA-B27 with AS and that HLA-B27 can serve as a marker for predisposition to diseases.

Keywords: Ankylosing spondylitis (AS); $\mathrm{Cy}-$ totoxic T cells (CTL); Heavy chain (HC); Human leukocyte antigen(s) (HLAs); Major histocompatibility complex (MCH); Relative risk (RR); Spondyarthropathies (SpA); T cell receptors (TCR).

\section{INTRODUCTION}

One of the most interesting areas of research within the field of histocompatibility concerns the observation that the major histocompatibility system is deeply involved in the development of a variety of diseases. Major histocompatibility complex (MHC) class I molecules are heterodimeric complexes consisting of a heavy chain (HC), $\beta 2$-microglobulin ( $\beta 2$ $\mathrm{m}$ or light chain), and a peptide (commonly 8-11 amino acids in length) that are located on the surface of most nucleated cells. Their biological function is to present peptides to a variety of ligands, in particular $\mathrm{T}$ cell receptors (TCR), residing on the surface of cytotoxic T cells (CTL) [1].

The human MHC class I molecule, human leukocyte antigen-B27 (HLA-B27), is strongly associated with spondyloarthropathies (SpA), a group of closely related inflammatory arthritic diseases, the most common of which is ankylosing spondylitis (AS) [1-3]. Spondyloarthropathies comprise several disorders that are more heterogenous clinically and where ge- 
netic susceptibility is likely to be more complex and variable [3]. The association degree varies markedly among different diseases of this group and also between different populations [2]. Association of AS and HLA-B27 remains one of the strongest known association of diseases with HLA-B27. About $95.0 \%$ of AS patients express HLA-B27, although the frequency of this antigen in Caucasians is below $10.0 \%$. However, the causative role of HLA-B27 in SpA is still not understood, although various studies confirmed that the HLA-B27 molecule itself is the strongest predisposing factor for disease pathogenesis [1].

A number of theories explaining the pathogenetic role of HLA-B27 have been proposed $[1,4]$. The theories proposed to explain the mechanism by which HLA-B27 induces disease susceptibility are: 1) HLA $B 27$ is in genetic linkage with a diseaseassociated gene; 2) HLA-B27 binds and presents "arthritogenic" peptides to T cells; 3) HLA-B27 is involved in thymic selection of a T-cell repertoire that is susceptible to SpA; 4) HLA-B27 has an unusual cell biology compared with other HLA class I molecules; 5) the HLA-B27 free cysteine at position $67 \mathrm{can}$ be chemically modified, leading to an "altered self;" 6) there is cross reactivity between antibodies directed at bacterial protein(s) and HLA-B27; 7) HLA-B27 is a receptor for a bacterial ligand; 8 ) interaction of HLA-B27 with bacterial superantigens causes non specific T-cell stimulation; 9) HLA-B27-derived peptides are presented by HLA class II molecules to $\mathrm{CD} 4^{+} \mathrm{T}$ cells [4-6]. None of the existing theories have as yet satisfactorily explained the underlying mechanism and the differential association of HLAB27 subtypes with AS. There are 86 HLA-B27 alleles based on nucleotide sequence differences, but at the translated protein level, the number of subtypes includes 81 different isoforms [7]. The common subtypes $\mathrm{B} * 27: 02, \mathrm{~B} * 27: 04$ and $\mathrm{B} * 27: 05$ are strongly associated with AS. Although some of the HLA-B27 subtypes, such as B*27:09 in Sardinia and B*27:06 in Thailand, have been clearly shown not to predispose to the disease $[1,3,8]$; sequencing of the HLA-B27 subtypes that are commonly associated with disease have shown no differences between healthy subjects and patients with AS. It is therefore believed that other genes and/or environmental factors are also involved in determining susceptibility to disease, and furthermore, that the degree of expression of the HLA-B27 gene may also be relevant [9].
The recent development of the genome-wide association study approach has revolutionized genetic studies of AS by finding some non MHC genes, some of which also confer susceptibility to psoriasis and chronic inflammatory bowel disease, such as the gene for the interleukin-23 (IL-23) receptor. The $I L-23 R$ gene has recently been found to contribute approximately $9.0 \%$ of the population- attributable genetic risk for AS in Caucasians. Another gene, ARTS1 (also called ERAAP and ERAP1) that encodes a trans membrane amino peptide with diverse immunological functions and is located on chromosome 5, shows strong association with AS, contributing roughly $23.0 \%$ of the population-attributable genetic risk for AS in Caucasian populations. The $I L-1$ gene cluster, IL $1 \mathrm{~A}$, contributes approximately $5.0 \%$ of the population-attributable genetic risk for AS in Caucasian populations. Thus, the popula-tion-attributable genetic risk for HLA-B27 (40.0\%), ARTS1 (23.0\%), IL-23R $(9.0 \%)$ and $I L-1 A(5.0 \%)$ add up, and one can conclude that approximately three-fourths of the total genetic risk of developing AS has been uncovered [8]. The aim of the present study was to determine the involvement of HLA-B27 in pathogenesis of AS, polyarthralgia, lumboishialgia, acute anterior uveitis (AAU), psoriatic arthritis (PA), synovitis coxae and rheumatoid arthritis (RA) in patients from Vojvodina, Serbia.

\section{MATERIALS AND METHODS}

Ninety-seven patients, of whom 58 were male and 39 were female, with a mean age of $39.6 \pm 14.4$ years, suffering from different diseases: AS (16), polyarthralgia (34), lumboishialgia (14), AAU (11), PA (3), synovitis coxae (16) and RA (3), composed of different ethnicities, residents of different parts of Vojvodina, were included in our study. In addition, a group of 224 healthy, unrelated potential bone marrow/organ donors, representing the same ethnic groups as the patients who resided in the same geographic area, served as a control population. An informed consent was obtained from all the individuals participating in the study and all institutional ethics requirements were met. Three mililiters of venous blood taken in vacutainers with EDTA as anticoagulant was collected from the patients and controls. Peripheral mononuclear cells were isolated by ficoll hypaque gradient centrifugation from which the $\mathrm{T}$ lymphocytes were separated by immunomagnetic beads (Dynabeads, 
Cat. No.: 219.02; Invitrogen, Oslo, Norway). The HLA class I typing was performed by serological immunomagnetic two-color fluorescence method using peripheral blood $\mathrm{T}$ lymphocytes and antigenspecific commercially available sera defining a single HLA specificity. Briefly, Terasaki microtiter plates (InnoTrain Diagnostik GmbH, Kronberg, Germany), containing various anti-HLA class I antisera were seeded with $1 \mu \mathrm{L}$ of a $2 \times 10^{6}$ cells $/ \mathrm{mL}$ suspension of immobilized T-cells. After incubation at room temperature and addition of $5 \mu \mathrm{L}$ rabbit complement, the lysed and vital lymphocytes were assessed using an ethidium bromide/acridine orange dye (Merck, Darmstadt, Germany) under an inverse phase contrast microscope $[10,11]$. Phenotype frequencies were obtained by a direct counting method, according to equation: $\mathrm{A}=n / \mathrm{N}$, where $n$ is number of persons with a given antigen and $\mathrm{N}$ is total number of persons studied. The strength of association of disease with respect to a particular HLAs is expressed by odds ratio (OR) interpreted as relative risk (RR). Relative risk is calculated for those HLAs that are increased or decreased in patients as compared to the control group. The RR or the number of times risk of disease is increased or decreased in individuals with a certain HLA marker, was calculated according to following formula

$$
R R=\frac{P^{+} x C^{-}}{C^{+} x P^{-}}
$$

where $\mathrm{P}^{+}$and $\mathrm{C}^{+}$are the number of patients or healthy persons who have a given antigen; $\mathrm{C}^{-}$and $\mathrm{P}^{-}$are the number of patients or healthy persons who do not have a given antigen, respectively $[12,13,14]$.

The values between 0 and 1 are of significance. When RR was higher than 1, we calculated an etiological fraction (EF) or population attributive risk, according to formula:

$$
E F=\frac{(F A D-F A P)}{(1-F A P)}
$$

where FAD is the frequency of the HLA-B27 antigen in the subgroup of patients and FAP is the HLA-B27 frequency in controls. The EF indicates the hypothetical genetic component of the disease and gives the proportion of disease cases attributable to a marker in the population (in this case, an HLA-B27 antigen), that presents a positive association $(\mathrm{RR}>1)$ with disease, and/or with other risk factors associated with this marker. Preventive fraction (PF) is calculated for negative association only where RR was lower than 1 , according to following equation:

$$
F P=\frac{(1-R R) x f}{R R x(1-f)+f}
$$

where $R R$ is relative risk and $f$ is the frequency of the HLA-B27 antigen in the subgroup of patients. The PF gives the percentage of cases that can be prevented if the population is exposed to an intervention compared to unexposed population. The EF and PF values greater than 0.15 were considered to reflect positive and negative association, respectively $[15,16]$. The $\chi^{2}$ test was performed to find out if there was a significant difference of HLA-B27 antigen frequency between controls and investigated subgroups of patients, according to following formula:

$$
X^{2}=\frac{(a d-b c)^{2}(a+b+c+d)}{(a+b)(c+d)(a+c)(b+d)}
$$

where a and $\mathrm{c}$ are the number of patients or healthy persons who have a given antigen; $b$ and $d$ are the number of patients or healthy persons who do not have a given antigen, respectively $[17,18]$.

\section{RESULTS}

Table 1 presents the distribution of HLA-B27 antigen in investigated subgroups of patients and controls from the Vojvodina population. It is noticeable that a significant increase in HLA-B27 frequency was found in the subgroup of patients with AS, $62.5 \% \mathrm{vs}$. $6.2 \%$ in controls, followed by the frequency of $18.1 \%$ in the patients with $\mathrm{AAU}, 12.5 \%$ in patients with synovitis coxae. The HLA-B27 frequency was similar in the patients with lumboischialgia (7.1\%) and polyarthralgia $(5.8 \%)$ compared to controls, while the frequencies found for the group of patients with PA and RA (33.3\% for both subgroups) cannot reflect the real situation due to the small number of patients included in this study.

Table 2 presents the result of calculated risk on disease susceptibility with HLA-B27. A relative risk higher than 1 was observed for subgroups of patients with AS $(\mathrm{RR}=25.0), \mathrm{PA}$ and $\mathrm{RA}(\mathrm{RR}=7.5$ for both subgroups), AAU ( $R R=3.33)$, synovitis coxae (RR $=2.142)$ and lumboishialgia $(\mathrm{RR}=1.153)$, but positive association (EF greater than 0.15 ) was found in subgroups of patients with AS $(E F=0.600)$. For PA and RA subgroups, $\mathrm{EF}=0.288$ for both of groups, 
Table 1. HLA-B27 antigen distribution among the investigated subgroups of patients and controls from Vojvodina, Serbia.

\begin{tabular}{|c|c|c|c|}
\hline Disease & HLA-B27 Positive (+) & HLA-B27 Negative $(\varnothing)$ & Frequency \\
\hline Ankylosing spondylitis & 10 & 6 & 0.625 \\
\hline Polyarthralgia & 2 & 32 & 0.058 \\
\hline Lumboishilagia & 1 & 13 & 0.071 \\
\hline Acute anterior uveitis & 2 & 9 & 0.181 \\
\hline Psoriatic arthritis & 1 & 2 & 0.333 \\
\hline Synovitis coxae & 2 & 14 & 0.125 \\
\hline Rheumatoid arthritis & 1 & 2 & 0.333 \\
\hline Controls $(n=224)$ & 14 & 210 & 0.062 \\
\hline
\end{tabular}

Table 2. Association between HLA-B27 and susceptibility to spondyloarthropathies in Vojvodina, Serbia.

\begin{tabular}{|c|c|c|c|c|c|}
\hline Disease & RR & EF & PF & $\chi^{2}$ & Association \\
\hline Ankylosing spondylitis & 25.0 & 0.6001 & - & $52.5^{\mathrm{a}}$ & positive \\
\hline Polyarthralgia & 0.9375 & - & 0.0666 & $1.75^{\mathrm{b}}$ & negative \\
\hline Lumboishilagia & 1.1530 & 0.0094 & - & $0.01^{\mathrm{b}}$ & negative \\
\hline Acute anterior uveitis & 3.33 & 0.1260 & - & $2.35^{\mathrm{b}}$ & negative \\
\hline Psoriatic arthritis & 7.5 & 0.2880 & - & $3.51^{\mathrm{b}}$ & positive \\
\hline Synovitis coxae & 2.142 & 0.0671 & - & $9.37^{\mathrm{a}}$ & negative \\
\hline Rheumatoid arthritis & 7.5 & 0.288 & - & $3.51^{\mathrm{b}}$ & positive \\
\hline
\end{tabular}

$\mathrm{V}$ (degree of freedom): 1; borderline value: 3.841 (corresponding to $p<0.05$ ); positive association: $\mathrm{EF}>0.15$; negative association: $\mathrm{PF}<0.15$.

${ }^{a}$ There was significant difference.

${ }^{\mathrm{b}}$ No significant difference.

but the very small number of patients was an obstacle for accurate statistical analysis.

A lower frequency of HLA-B27 antigen compared to the control group was found in the subgroup of patients with polyarthralgia $(\mathrm{RR}=0.9375)(\mathrm{PF}$ is lower than 0.15 ), but negative association was not statistically significant ( $\chi^{2}$ is not greater than 3.841). The $\chi^{2}$ test showed the significant difference between HLA-B27 frequencies in the subgroups of patients with ankylosing spondylitis $\left(\chi^{2}=52.5\right)$ and synovitis coxae $\left(\chi^{2}=9.37\right)$, in comparison to controls. The $\chi^{2}$ values exceeding 3.841 (corresponding to $p<0.05$ ) were regarded as a stable borderline index for significant differences between HLA-B27 frequencies in controls and investigated subgroups of patients.

\section{DISCUSSION}

The genetic susceptibility of a population to a particular disease can be known through the study of HLA genetics. As the genes come from paren- tal segregation, genetic screening can predict the risk of development of the disease, and the disease can be detected before onset or at a very early stage $[4,9,19,20]$. It has now been 35 years since the association of HLA-B27 and AS has been demonstrated. Over $90.0 \%$ of patients with AS express the HLAB27 antigen, but only $1.0-6.0 \%$ of HLA-B27 positive individuals in the general population develop AS $[4,19]$. In contrast, in families with AS, the prevalence is $10.0-30.0 \%$ in adult first-degree relatives inheriting HLA-B27. The concordance in identical twins is approximately $65.0 \%$. It is currently believed that susceptibility to AS is determined almost entirely by genetic factors, with HLA-B27 comprising about one-third of the genetic component $[3,21,22]$.

In the present study we have demonstrated for the first time the distribution of HLA-B27 in SpA patients of Vojvodina ethnicity. For the whole SpA group, we have found 19 of 97 patients to be HLA-B27 positive (19.58\%). The highest frequency of HLA-B27 (62.5\%) was observed in AS patients, following with 
the $33.3 \%$ in both of groups of patients with psoriatic arthritis as well as rheumatoid arthritis, respectively. Acute anterior uveitis and synovitis coxae patients revealed lower frequencies of the investigated marker, 18.1 and $12.5 \%$, respectively. In the group of patients with lumboihialgia and polyarthralgia, there were 7.1 and $5.8 \%$ HLA-B27 positive patients. In the control group of healthy subjests from Vojvodina, the HLA- B27 marker was detected in 14 of 224 individuals (6.2\%). In the AS group, our findings in the present study corroborate other studies, which point to a strong association between HLA-B27 and AS $[2,8,23,24]$. The distribution of HLA-B27 in our sample of AS patients from Vojvodina is similar from that found in populations of Tunisia, $(62.0 \%)$, Italy (76.0-68.0\%) and Turkey (70.0\%) [2,21]. In contrast, the HLA-B27 frequency in AS patients from Vojvodina was lower than that reported from different European countries: Spain-Galicia (94.3\%), United Kingdom (90.2-94.0\%), Norway (north) (93.0\%), Finland (93.0\%), Hungary (92.7\%), Bulgaria (88.0\%), Spain (84.4\%), Germany (82.0\%) and Romania (72.1\%) [2]. Since the number of our RA and PA patients was small, the HLA-B27 frequency (33.3\%) as well as $(R R=7.5$ and $E F=0.288)$, values we found may not reflect the real situation, as implied also by the suboptimal statistical power of this part of the study. However, as described in previous studies, HLA-B27 is detected with 5-10 times greater frequency in RA patients than in the general population $[2,25,26]$, and the frequency of this marker in our study compared to reports of some studies, was similar [2,27]. Also, the HLA-B27 frequency in the investigated patients with PA we found in our study was higher than that reported from Ireland (15.0\%), continental Italy $(12.4 \%)$ and central Serbia (7.0\%) [2,27], but closer to the frequencies reported from the United Kingdom (26.0\%) and Spain (22.0\%) [2,28]. In the AAU group, the HLA-B27 frequency in our Vojvodina sample was higher than that reported for the Tunisian patients (4.4\%) [29] and Asia (6.0-13.0\%), but lower than that reported for western countries (18.0-32.0\%) [30,31] and Indian population (56.2\%) [32].

Although, there was a statistical difference in HLA-B27 frequency in the subgroup of patients with synovitis coxae in comparison to controls and RR was 2.142 , there is no positive association of synovitis coxae and HLA-B27 antigen in the population of Vojvodina $(\mathrm{EF}=0.0671)$. In other investigated subgroups of patients, such as for patients with lumboishialgia and acute anterior uveitis, RR was higher than 1, but there is no positive association with HLAB27 since EF were 0.0094 and 0.1260 , respectively.

\section{CONCLUSIONS}

In conclusion, our study revealed that HLAB27 was more common in patients with AS than in the general population of Vojvodina, Serbia. These findings suggest that HLA typing would help in the diagnosis of spondyloarthropathies, specially AS, as well as identifying at-risk family members.

\section{REFERENCES}

1. Hulsmeyer M, Hillig RC, Volz A, Ruhl M, Schroder W, Saengen W, et al. HLA-B27 Subtypes differentially associated with disease exhibit subtle structural alterations. J Biol Chem. 2002; 227(49): 47844-47853.

2. Popa OM, Bojinica M, Bojinica V, Ciofu C, Dutescu MI, Bardan A, et al.Distribution of HLAB27 in Romanian spondyloarthritides patients. Int J Immunogenet. 2010; 37(6): 513-516.

3. Colbert RA, DeLay ML, Layh-Schmitt G, Sowders DP. HLA-B27 misfolding and spondyloarthropathies. Prion. 2009; 3(1): 15-26.

4. Bowness P. HLA B27 in health and disease: a double-edged sword? Rheumatology. 2002; 41(8): 857-868.

5. Huhtinen M. Acute anterior uveitis and HLAB27: infectious background, systemic inflamation and prognosis of the patients. Academic dissertation, defended at the Medical Faculty of the University of Helsinki, Helsinki, 2002.

6. Allen RL, Bowness P, McMichael AJ. The role of HLA-B27 in spondyloarthritis. Immunogenetics. 1999; 50(3-4): 220-227.

7. Marsh SGE. Nomenclature for factors of the HLA system, update September 2011. Int J Immunogenet. 2011; 39(1): 95-97.

8. Khan MA. Recent advances in the genetics of ankylosing spondylitis. Future Rheumatol. 2008; 3(5): 403-405.

9. Cauli A, Dessole G, Fiorillo MT, Vacca A, Mameli A, Bitti P, et al. Increased level of HLA- 
B27 expression in ankylosing spondylitis patients compared with healthy HLA-B27-positive subjects: a possible further susceptibility factor for the development of disease. Rheumatology. 2002; 41(12): 1375-1379.

10. Vartdal F, Gaudernack G, Funderud S, Bratlie A, Lea T, Ugelstad J, et al. HLA class I and II typing using cells positively selected from blood by immunomagnetic isolation-a fast and reliable technique. Tissue Antigens. 1986; 25(5): 301-312.

11. Vartdal F, Bratlie A, Gaudernack G, Funderud S, Lea T, Thorsby E. Microcytotoxic HLA typing of cells directly isolated from blood by means of antibody-coated microspheres. Transplant Proceedings. 1987; 19(1): 655-657.

12. Walker RH. Technical Manual, 11th ed. Bethesda: American Association of Blood Banks, 1993: 305-307.

13. Stites DP, Stobo JD, Wells JV. Osnovna i klinička imunologija. Beograd: Savremena Administracija, 1989; 50-63.

14. Svejgaard A, Platz R, Ryder LP. HLA and disease 1982-A survey. Immunolog Rev. 1983; 70(1): 193-201.

15. Bengston BO, Thompson G. Measuring the strength of associations between HLA antigens and disease. Tissue Antigens. 1981; 18(5): 356-363.

16. Green A. The epidemiologic approach to studies of association between HLA and disease.II Estimation of absolute risk, etiologic and preventive fraction. Tissue Antigens. 1982; 19(4): 259-168.

17. Petz B. Osnovne statističke metode. Zagreb: Izdanja škole narodnog zdravlja "Andrija Štampar." 1967: 111-134.

18. Snedecor GW, Cochan GW. Statistical Methods. Ames: The Iowa State University Press. 1956: 219-221.

19. Emam SJ, Badri S, BayatA, Dashtebozorg N, Khosravi A. Evaluation of 278 HLA-B27 positive patients suspected of seronegative spondyloarthropathies. Pakistan J Med Sci. 2007; 23(5): 728-732.

20. McMichael A, Bowness P. HLA-B27:natural function and pathogenic role in spondyloarthritis. Arthritis Res. 2002; 4(Suppl 3): S153-S158.
21. Sahin S, Aydogan L, Benli I, Ozyurt H. Distribution of HLA-B27 and CYP2D6*4 mutations in the middle Black Sea area (Tokat) of Turkey. Genet Molec Res. 2011; 10(4): 3987-3991.

22. Sheehan NJ. The ramifications of HLA-B27. J Roy Soc Med. 2004; 97(1): 10-14.

23. Dhurandhar PS, Shankarkumar U. HLAAssociation in seronegative spondyloarthritis patients from Mumbai, India. Int J Hum Genet. 2007; 7(3): 235-239.

24. Pitchappan RM, Balakrlshnan K, Mahendran V, Brahmajathi V. HLA and disease susceptibility in Tamil Nadu, S. India. Indian J Hum Genet. 1995; 1(1):56-62.

25. Kim T-H, Uhm W-S, Inman RD. Pathogenesis of ankylosing spondylitis and reactive arthritis. Curr Opin Rheumatol. 2005; 17(4): 400-405.

26. Colmegna I, Cuchacovich R, Espinoza LR. HLA- B27 associated reactive arthritis: pathogenetic and clinical considerations. Clin Microbiol Rev. 2004; 17(2): 348-369.

27. Carter JD, Hudson AP. Reactive arthritis: clinical aspects and medical management. Rheumatic Dis Clin North Am. 2009; 35(1): 21-44.

28. FitzGerald O, Winchester R. Psoriatic arthritis: from pathogenesis to therapy. Arthritis Res Ther. 2009; 11(1): 214-223.

29. Khairallah M,Yahia SB, Ladjimi A, Messaoud $\mathrm{R}$, Zaouali S, Attia S, et al. Pattern of uveitis in a referral centre in Tunisia, North Africa. Eye. 2007; 21(1): 33-39.

30. Chang JH, McCluskey PJ, Wakefield D. Acute anterior uveitis and HLA-B27: what's new? In: Pleyer U, Forrester JV, Eds. Uveitis and Immunological Disorders. Heidelberg: SpringerVerlag. 2009:9-17.

31. Chang JH, McCluskey PJ, Wakefield D. Acute anterior uveitis and HLA-B27. Survey Ophtalmol. 2005; 50(4): 364-388.

32. Mishra MN, Bharucha KM. HLA-B27 Association with uveitis in an Asian Indian population. Iranian J Immunol. 2011; 8(2): 85-89. 